
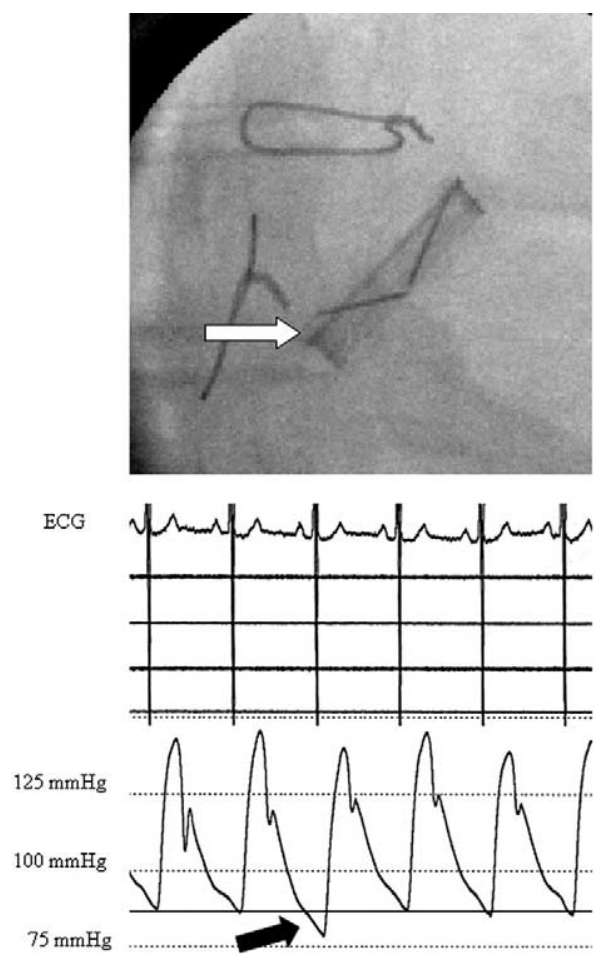

Figure 1. Fluoroscopy of an ADVANTAGE bileaflet valve in the aortic position and recording of the invasive blood pressure measurement in the ascending aorta with electrocardiography (ECG). While one leaflet is completely closed, the second leaflet stays in a slightly open position during the whole diastole (white arrow). The housing and leaflet edges are radiolucent and cannot be visualized with this technique. Diastolic blood pressure decrease is associated with each pathologic leaflet motion (black arrow).

\title{
Complete excision of primary cardiac malignant fibrous histiocytoma involving the left atrial free wall and mitral annulus by modified autotransplantation
}

Chih-Hsien Wang, MD, ${ }^{a}$ Hsi-Yu Yu, MD, ${ }^{a}$ Nai-Shin Chi, MD, ${ }^{a}$ Yih-Sharng Chen, MD, ${ }^{a}$ Kun-Kuang Lee, MD, ${ }^{a}$ Ya-Jung Cheng, MD, ${ }^{\text {b }}$ Lung-Chun Lin, MD, ${ }^{c}$ Wen-Yih Isaac Tseng, MD, PhD, ${ }^{d}$ and Shoei-Shen Wang, MD, PhD, ${ }^{a}$ Taipei, Taiwan

A

44-year-old male patient was referred to our hospital for progressive dyspnea on exertion and a cardiac tumor disclosed by a computed tomographic image study (Figure 1, A). Operative exploration was per-

From the Departments of Surgery, ${ }^{\mathrm{a}}$ Anesthesiology, ${ }^{\mathrm{b}}$ Medicine, ${ }^{\mathrm{c}}$ and Medical Imaging, ${ }^{\mathrm{d}}$ National Taiwan University Hospital and National Taiwan University College of Medicine, Taipei, Taiwan.

Received for publication Nov 3, 2005; revisions received Nov 15, 2005; accepted for publication Nov 18, 2005.

Address for reprints: Hsi-Yu Yu, MD, Department of Surgery, National Taiwan University Hospital, No 7, Chung-Shan S Rd, Taipei, Taiwan (E-mail: hsiyuyu@ha.mc.ntu.edu.tw).

J Thorac Cardiovasc Surg 2006;131:731-3

$0022-5223 / \$ 32.00$

Copyright $\odot 2006$ by The American Association for Thoracic Surgery doi:10.1016/j.jtcvs.2005.11.020 formed 3 days later, in which a wide-based tumor infiltrating the left atrial appendage, left atrial anterior free wall, anterior annulus of the mitral valve, and anterior mitral leaflet was found. Tumor debulking and removal of the left atrial appendage were performed to relieve obstruction by the tumor. Malignant fibrous histiocytoma was documented by microscopic examination. Follow-up computed tomography performed before his first hospital discharge revealed residual tumor existing at the anterior atrial wall.

The tumor grew fast, for 30 days, to reach a diameter of $3.6 \times$ $3.6 \mathrm{~cm}^{2}$ at his second admission for recurrent dyspneic symptoms. During the investigation process for possible cardiac allotransplantation, he suddenly experienced shortness of breath and collapsed in the ward because of nearly total obstruction of the mitral orifice by the rapid-growing tumor mass. Extracorporeal membrane oxygenation (ECMO) was set up on an emergency basis under cardiopulmonary resuscitation, and he was immediately sent to the operating room for a radical surgical procedure as his last chance. 

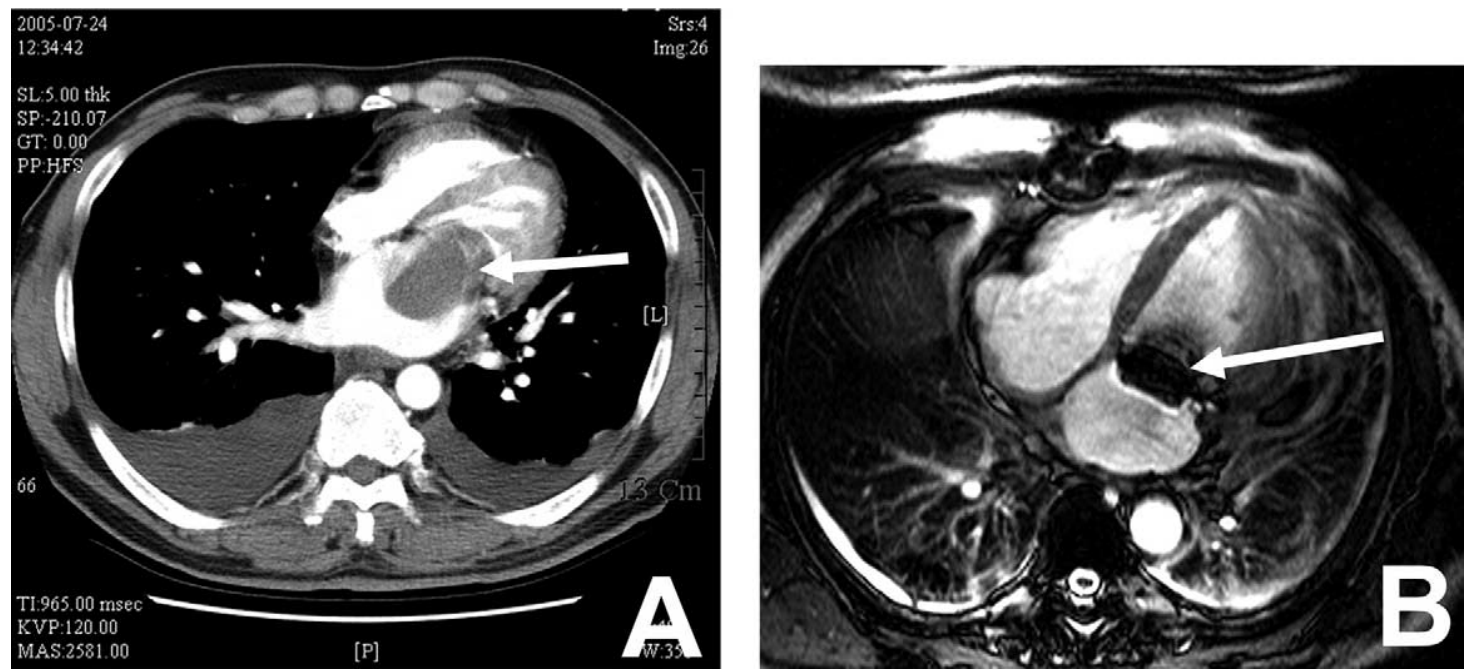

Figure 1. A, A $5 \times 7-\mathrm{cm}^{2}$ mass (arrow) in the left atrium adjacent to the anterior annulus of the mitral valve with a bilateral pleural effusion was found at the patient's first admission. B, Thirty-eight days after radical tumor excision and insertion of a prosthetic mitral valve (arrow), no evidence of tumor recurrence was found in the left atrium.

ECMO was shifted to standard cardiopulmonary bypass, and the chest was opened. After cold cardioplegic cardiac arrest, the heart was explanted with an atrial incision line made at both pulmonary venous cuffs. Multiple microscopic examinations of frozen sections of tissues from pulmonary venous cuff edges were made to ensure that no residual malignant cells remained. Pulmonary venous confluents were reconstructed with in situ pericardium, a modification from the procedure of reoperation for restenosis of total pulmonary venous return. ${ }^{1}$ The inferior vena cava was extended with a 20 -mm polytetrafluoroethylene (Gore-Tex; W. L. Gore \& Associates, Inc, Flagstaff, Ariz) tube graft. On the ex vivo cardiac side, first, the left main and circumflex coronary arteries and their concomitant cardiac veins were dissected to prevent later injury (Figure 2). Second, almost $240^{\circ}$ circumferentially except for the posterior atrial tissue was excised to achieve a wide tumor-free margin. The dissection plane went deep to include all the fibrous tissue of the anterior mitral annulus with a partial left ventriculectomy. The partially excised ventricular muscle was reinforced with transmural polytetrafluoroethylene strips to prevent left ventricular rupture after the operation. ${ }^{2}$ The atrial wall was reconstructed with equine pericardium, and a mechanical valve ( $\mathrm{St}$ Jude Medical, 29 mm; St Jude Medical, Inc, St Paul, Minn) was placed at the mitral position. The heart was then implanted back in usual fashion, and it resumed sinus rhythm thereafter. The total cardiopulmonary bypass time was 340 minutes, and crossclamp time was 220 minutes.

Delayed sternal closure was performed 3 days later. ECMO was continuously used for 5 days after the operation because of right heart failure. After gradual recovery of his liver, kidney, brain, heart, and respiratory function, he was transferred to the ward 30 days after surgery and discharged 38 days after surgery in stable condition. Follow-up magnetic resonance imaging performed before hospital discharge revealed no tumor recurrence. At 3 months of outpatient follow-up, the patient was well, without evidence of recurrence.

\section{Discussion}

Primary cardiac malignant fibrous histiocytoma was reported with a high local recurrence rate and a pessimistic prognosis. ${ }^{3}$ Cardiac allotransplantation might be an option for this disease. ${ }^{4}$ However, considering the rapid-growing nature of this tumor, from our experience with this case and from a literature review, ${ }^{4}$ in addition to relatively scarce heart resources for the large number of patients with class $1 \mathrm{~A}$ disease on a waiting list for heart transplantation, the patient's condition may deteriorate before a suitable donor heart is available, as in the presented case.

The surgical technique of cardiac autotransplantation was described clearly in an early report. ${ }^{5}$ For this case, we shifted the left atrial excision line from the atrial wall anterior to the pulmonary veins to the bilateral pulmonary venous cuffs and reconnected both pulmonary cuffs with in situ pericardium. ${ }^{1}$ This procedure facilitates more confidence in complete excision of malignant tumors, and it also lengthens the left atrial cuff to make atrial anastomosis easier.

In conclusion, we report a successful experience of aggressive treatment of primary malignant fibrous histiocytoma involving the left atrium and mitral valve by extensive tumor excision with a modified cardiac autotransplantation technique.

We are grateful to $\mathrm{C}$. W. Tsui for artistic assistance. 


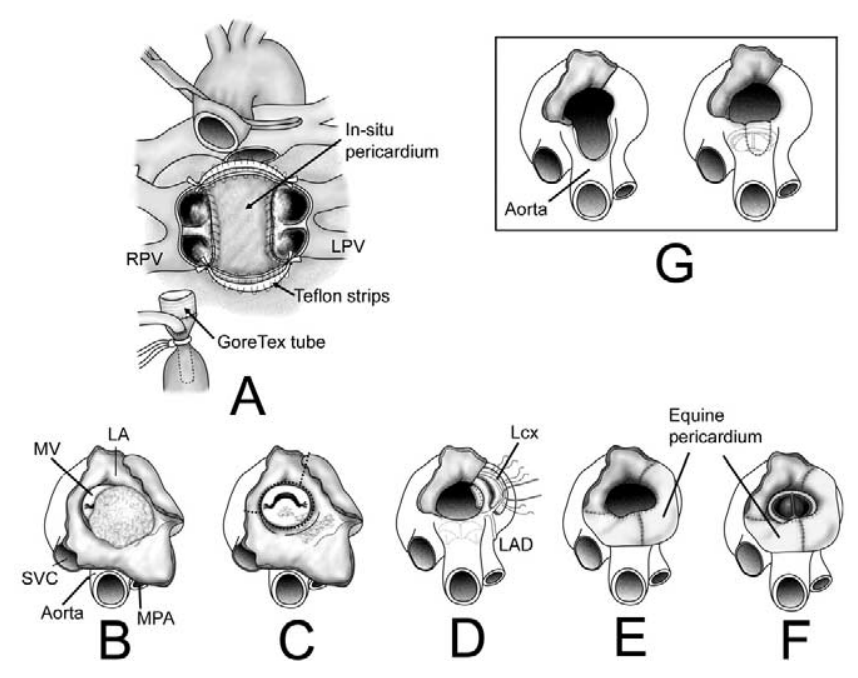

Figure 2. Illustration of operative procedures for tumor excision and reimplantation. A, Pulmonary venous confluents were reconstructed with in situ pericardium with Teflon (DuPont, Wilmington, Del) strip reinforcement. The inferior vena cava was extended with a $20-\mathrm{mm}$ polytetrafluoroethylene tube graft. B, The heart was explanted and turned over to reveal a huge tumor mass obstructing the mitral orifice. C, After removal of the main tumor mass, residual tumor involving the mitral anterior leaflet, annulus, and left atrial anterior wall was found. D, To achieve radical excision, the left main and circumflex coronary arteries and their concomitant cardiac veins were mobilized, and then nearly two thirds of the left atrial tissue, mitral anterior annulus and leaflet, and adjacent ventricular muscles was removed. The partially excised myocardium was reinforced with transmural polytetrafluoroethylene strips. $E$, The left atrial wall was reconstructed with equine pericardium. F, A 29-mm mechanical valve prosthesis was implanted. G, In case aortic annulus was also involved, the partial aortic wall and aortic valvular structure would also have been excised, followed by the insertion of an aortic mechanical valve and reconstruction of the aortic wall. $R P V$, Right pulmonary vein; $L P V$, left pulmonary vein; $M V$, mitral valve; $L A$, left atrium; SVC, superior vena cava; MPA, main pulmonary artery; $L c x$, left circumflex coronary artery; $L A D$, left anterior descending coronary artery.

\section{References}

1. Lacour-Gayet F, Zoghbi J, Serraf AE, Belli E, Piot D, Rey C, et al. Surgical management of progressive pulmonary venous obstruction after repair of total anomalous pulmonary venous connection. $J$ Thorac Cardiovasc Surg. 1999;117:679-87.

2. Campanella C, Sinclair C, Cameron E. Explantation, repair, and reimplantation of the heart for left ventricular rupture after mitral valve replacement. J Heart Lung Transplant. 1993;12(1 Pt 1):150-1.
3. Sabesan T, Xuexi W, Yongfa Q, Pingzhang T, Ilankovan V. Malignant fibrous histiocytoma: outcome of tumours in the head and neck compared with those in the trunk and extremities. Br J Oral Maxillofac Surg. In press.

4. Akhter SA, McGinty J, Konys JJ, Giesting RM, Merrill WH, Wagoner LE. Recurrent primary cardiac malignant fibrous histiocytoma following orthotopic heart transplantation. J Heart Lung Transplant. 2004;23:1447-50.

5. Conklin LD, Reardon MJ. Autotransplantation of the heart for primary cardiac malignancy: development and surgical technique. Tex Heart Inst J. 2002;29:105-8. 